\title{
Development of graphene process control by industrial optical spectroscopy setup
}

Fursenko, O., Lukosius, M., Lupina, G., Bauer, J., Villringer, C., et al.

O. Fursenko, M. Lukosius, G. Lupina, J. Bauer, C. Villringer, A. Mai, "Development of graphene process control by industrial optical spectroscopy setup," Proc. SPIE 10330, Modeling Aspects in Optical Metrology VI, 1033017 (26 June 2017); doi: 10.1117/12.2269603

SPIE. Event: SPIE Optical Metrology, 2017, Munich, Germany 


\title{
Development of graphene process control by industrial optical spectroscopy setup
}

\author{
O. Fursenko*a , M. Lukosius ${ }^{\mathrm{a}}$, G. Lupina ${ }^{\mathrm{a}}$, J. Bauer ${ }^{\mathrm{b}}$, C. Villringer $^{\mathrm{b}}$, A. Mai $^{\mathrm{a}}$ \\ ${ }^{a}$ IHP, Im Technologiepark 25, 15236 Frankfurt (Oder), Germany \\ ${ }^{\mathrm{b}}$ Technical University of Applied Sciences, Hochschulring 1, 15745 Wildau, Germany
}

\begin{abstract}
The successful integration of graphene into microelectronic devices depends strongly on the availability of fast and nondestructive characterization methods of graphene grown by CVD on large diameter production wafers [1-3] which are in the interest of the semiconductor industry. Here, a high-throughput optical metrology method for measuring the thickness and uniformity of large-area graphene sheets is demonstrated. The method is based on the combination of spectroscopic ellipsometry and normal incidence reflectometry in UV-Vis wavelength range $(200-800 \mathrm{~nm})$ with small light spots $\left(\sim 30 \mu \mathrm{m}^{2}\right)$ realized in wafer optical metrology tool. In the first step graphene layers were transferred on a $\mathrm{SiO}_{2} / \mathrm{Si}$ substrate in order to determine the optical constants of graphene by the combination of multi-angle ellipsometry and reflectometry. Then these data were used for the development of a process control recipe of CVD graphene on 200 $\mathrm{mm} \mathrm{Ge}(100) / \mathrm{Si}(100)$ wafers. The graphene layer quality was additionally monitored by Raman spectroscopy. Atomic force microscopy measurements were performed for micro topography evaluation. In consequence, a robust recipe for unambiguous thickness monitoring of all components of a multilayer film stack, including graphene, surface residuals or interface layer underneath graphene and surface roughness is developed. Optical monitoring of graphene thickness uniformity over a wafer has shown an excellent long term stability $(s=0.004 \mathrm{~nm})$ regardless of the growth of interfacial $\mathrm{GeO}_{2}$ and surface roughness. The sensitivity of the optical identification of graphene during microelectronic processing was evaluated.

This optical metrology technique with combined data collection exhibit a fast and highly precise method allowing one an unambiguous detection of graphene after transferring as well as after the CVD deposition process on a $\mathrm{Ge}(100) / \mathrm{Si}(100)$ wafer. This approach is well suited for industrial applications due to its repeatability and flexibility.
\end{abstract}

Keywords: graphene, spectroscopic ellipsometry, reflectometry, optical metrology, AFM

\section{INTRODUCTION}

The successful integration of graphene into microelectronic devices depends not only on various technological aspects, but also on the availability of fast and nondestructive characterization methods of graphene grown by CVD on large diameter production wafers [1-3] which are in the interest of the semiconductor industry.

To date the dominant microelectronic technology is based on Si and enabling its new functionalities by adding graphene-based devices is considered as a very appealing scenario. However, the formation of carbide during the growth process makes the fabrication of graphene directly on $\mathrm{Si}$ wafers extremely challenging. To overcome these difficulties and to reach the target of properties, in [2-3] growth technique of high quality graphene layers by the CVD method on $\mathrm{Ge}(100) / \mathrm{Si}(100)$ wafers was proposed. The availability of smooth, more than $2 \mu \mathrm{m}$ thick $\mathrm{Ge}(100)$ epi layers with a low threading dislocation density on $\mathrm{Si}(100)$ [4] enables such fabrication of graphene layers. The characterization of CVD graphene grown on $\mathrm{Ge}(100 / \mathrm{Si}(100)$ is of interest to the semiconductor industry because CVD graphene allows for the fabrication of devices across large diameter wafers in contrast to small configurations (few hundred $\mu \mathrm{m}^{2}$ ) of mechanically exfoliated flakes.

*fursenko@ihp-microelectronics.com; phone (+49) 3355625 328; ihp-microelectronics.com

Modeling Aspects in Optical Metrology VI, edited by Bernd Bodermann, Karsten Frenner,

Richard M. Silver, Proc. of SPIE Vol. 10330, 1033017 · (c) 2017 SPIE

CCC code: $0277-786 \mathrm{X} / 17 / \$ 18 \cdot$ doi: $10.1117 / 12.2269603$

Proc. of SPIE Vol. $103301033017-1$ 
The characterization of graphene films deposited on substrates different from $\mathrm{SiO}_{2} / \mathrm{Si}$ has been a challenging task so far. Characterization of films less than $2 \mathrm{~nm}$ on bulk substrates using conventional reflectometry techniques as it was proposed for exfoliated graphene on $\mathrm{SiO}_{2}$ [5] is an insoluble task. With decreasing thickness, the optical response of the film is diminishing and the small signal changes are often buried in the noise. When graphene is deposited directly on $\mathrm{Ge} / \mathrm{Si}$ substrate, reflectometry cannot provide enough sensitivity for adequate film characterization. In this case spectroscopic ellipsometry can be a good solution.

This study is based on the investigation of graphene layers transferred on $\mathrm{SiO}_{2} / \mathrm{Si}$ substrate in order to determine the optical constants of graphene by the combination of multi-angle ellipsometry and multi-angle reflectometry. Once the optical properties of graphene are accurately determined, the graphene monolayer films can be detected with confidence on almost any substrate, for example on $\mathrm{Ge} / \mathrm{Si}$. The sensitivity of spectroscopic ellipsometry and reflectometry for in-line graphene deposition process control on $\mathrm{Ge}(100) / \mathrm{Si}(100)$ wafer is investigated. We will show that CVD graphene deposition process can be reliably evaluated by the combination of spectroscopic ellipsometry and reflectometry on wafer level tool with unambiguous thickness monitoring of all components of a multilayer film stack, including graphene, surface residuals or interface layer underneath graphene and surface roughness.

\section{EXPERIMENTAL}

Graphene was deposited by CVD on 200-mm Ge(100)/Si(100) wafers in Aixtron's Black Magic BM300T CVD tool at $885^{\circ} \mathrm{C}$ using $\mathrm{CH}_{4}$ as source of carbon and $\mathrm{Ar} / \mathrm{H}_{2}$ mixture as carrier gas. The pressure of 700 mbar was kept during the depositions. The optimized deposition time was $60 \mathrm{~min}$. The details of the growth of these films are published in [6-7]. Epitaxial $\mathrm{Ge}(100)$ with a low threading dislocation density (TDD) (below $1 \times 10^{8} \mathrm{~cm}^{-2}$ ) and low surface roughness, deposited by reduced pressure chemical vapor deposition (RPCVD) [4] on Si(100) wafers, was used as a substrate. A $2 \mu \mathrm{m}$-thick Ge layer was selected in order to prevent any Si diffusion in the Ge surface at high temperature.

For optical constant model development Graphene flakes of several square centimeters were transferred by a wettransfer technique on $\mathrm{Si}(100)$ wafer covered with $\mathrm{SiO}_{2}(\sim 300 \mathrm{~nm}$ thick). Poly- (mehyl methacrylate) (PMMA) was used as a graphene carrier.

Optical spectroscopic measurements were performed on the KLA-Tencor Spectra Fx200 wafer metrology tool using a combination of spectroscopic ellipsometry (SE) with $\sim 71^{\circ}$ angle of incidence (AOI) in the wavelength range of 240$800 \mathrm{~nm}$ and with $\sim 63^{\circ} \mathrm{AOI}$ in the $200-300 \mathrm{~nm}$ range, and reflectometry (R) in the range of 200-800 nm with near normal incidence $\left(\mathrm{AOI}=1.66^{\circ}\right)$ for determination of thickness, optical constants and roughness of graphene films. Small spot objectives $(30-50 \mu \mathrm{m})$ were used. Additionally, the multi-angle ellipsometry (AOI=50, 60 ${ }^{\circ}$ ) (SENresearch SE800 DUV

2C 16M (SENTECH)) with spot size of $1 \mathrm{~mm}$ and multi-angle reflectometry (AOI=20, 30 ${ }^{\circ}$ (Lambda $1050 \mathrm{UV} / \mathrm{Vis} / \mathrm{NIR}$ (Perkin Elmer)) with spot size of $\sim 4 \mathrm{~mm}$ were carry out on transferred graphene on $\mathrm{SiO}_{2} / \mathrm{Si}$. Combined data collection was chosen for unambiguous determination of graphene optical constants.

Raman spectroscopy was applied for graphene quality layer evaluation. The room temperature measurements were performed using the Renishaw In-Via system. The excitation source was a coherent mixed $\mathrm{Ar}+/ \mathrm{Kr}+$ ion gas laser at $\lambda=514.5 \mathrm{~nm}$. The laser power applied to the sample was about $1 \mathrm{~mW}$ with $50 \mathrm{x}$ microscope objective, which prevents any sample degradation. The laser spot diameter on the wafer surface was $\sim 0.5 \mu \mathrm{m}$. Atomic force microscopy (AFM) measurements with Park NX20 system were performed for micro topography evaluation.

\section{RESULTS AND DISCUSSION}

\subsection{Optical constants determination for $\mathrm{Gr} / \mathrm{SiO}_{2} / \mathrm{Si}(100)$ film stack}

The detection of monolayer of graphene by ellipsometry or reflectometry requires the optical constants of graphene. They should be carefully determined to enable optical methods such as spectroscopic ellipsometry and reflectometry for material quality evaluation and process monitoring. In order to determine the optical constants precisely, a suitable film stack is needed. The best choice is a $\mathrm{Gr} / \mathrm{SiO}_{2} / \mathrm{Si}$ stack where graphene $(\mathrm{Gr})$ is transferred by either electrochemical delamination or growth catalyst etching on $\mathrm{SiO}_{2} / \mathrm{Si}$ [8]. The $\mathrm{SiO}_{2}$ layer with a thickness of about $300 \mathrm{~nm}$ causes a good optical contrast due to a significant interference effect and can improve the accuracy of optical constants and thickness determination. The transfer process is one of the crucial steps since, if not performed properly, it can greatly decrease the quality of the graphene layer. The Raman spectroscopy measurements (not shown here) have confirmed the existence of 
a monolayer of graphene with good quality [7]. In order to reduce possible errors for determination of optical constants the combination of multi-angle ellipsometry and reflectometry data was applied for the fitting procedure. The optical constants of graphene were extracted by a point-by-point fit at each individual wavelength. For monolayer graphene, since anisotropy cannot be resolved, an isotropic model is used. In Figure 1 the extracted optical constants are shown.

A pronounced peak is observed for the ultraviolet adsorption of graphene similar to that reported in $[9,10]$. Its asymmetric shape and the peak position at $5.01 \mathrm{eV}$ corresponds to the saddle point and is attributed to a van Hove singularity in the density of states based on three-dimensional interband critical point analysis and modifies by a strong excitonic effect with an exciton absorption peak at $4.55 \mathrm{eV}$, obtained by application of a Lorentz oscillator model, that it was established from the measured optical constants.

These optical constants were applied further for the identification and determination of the thickness of graphene on $\mathrm{Ge}(100) / \mathrm{Si}(100)$ substrates.

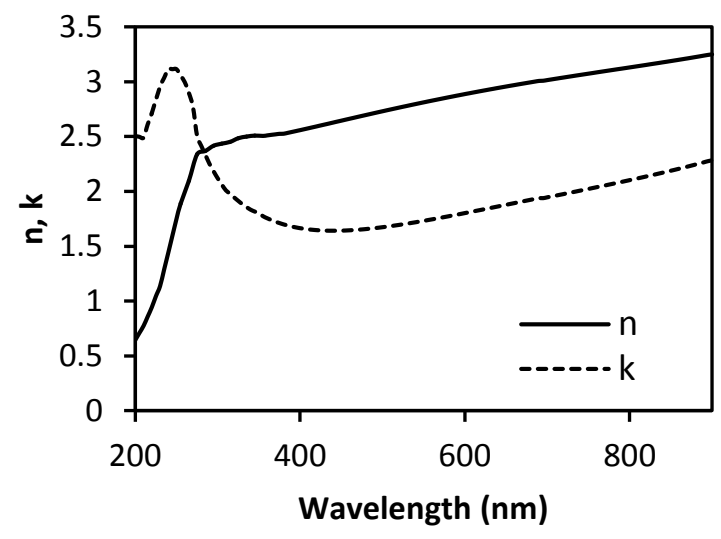

Figure 1. Optical constants of graphene.

\subsection{Process control of $\mathrm{Gr} / \mathrm{Ge}(100) / \mathrm{Si}(100)$}

High quality graphene was grown on $\mathrm{Ge}(100) / \mathrm{Si}(100)$ substrates which was confirmed by the results of Raman spectroscopy measurements. Figure 2 shows a Raman spectrum acquired along the $200 \mathrm{~mm} \mathrm{Gr} / \mathrm{Ge}(100) / \mathrm{Si}(100)$ wafer diameter showing characteristic G, 2D, and D bands of graphene. Raman spectra confirmed that the samples were singlelayer graphene. Strong G and 2D Raman bands and a weak D peak confirm that good quality graphene is formed.

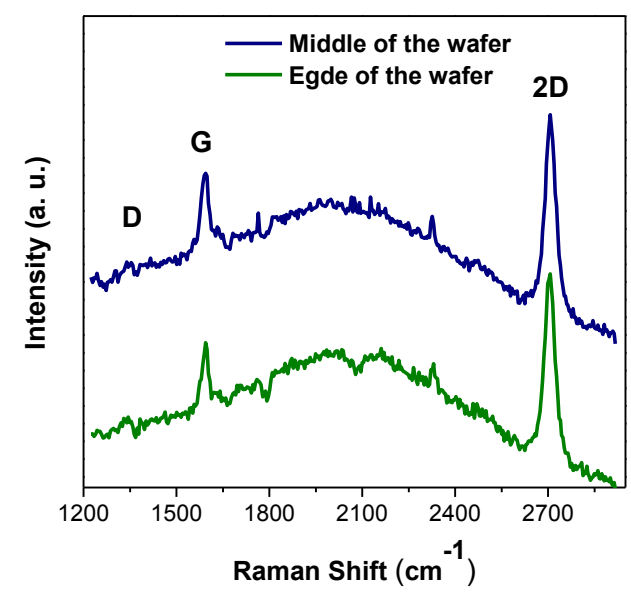

Figure 2. Raman spectra acquired along the $200 \mathrm{~mm} \mathrm{Gr} / \mathrm{Ge}(100) / \mathrm{Si}(100)$ wafer diameter showing characteristic G, 2D, and D bands of graphene. 
The surface structure of deposited graphene was proven by AFM measurements. Figure 3 shows the AFM topography images of the surfaces before (a) and after (b) graphene deposition on $\mathrm{Ge}(100) / \mathrm{Si}(100)$ for $2 \times 2$ and $30 \times 30 \mu \mathrm{m}$ scan sizes. The increase of the mean square surface roughness $\sigma$ is observed after graphene deposition as it was also demonstrated in [6].

(a)
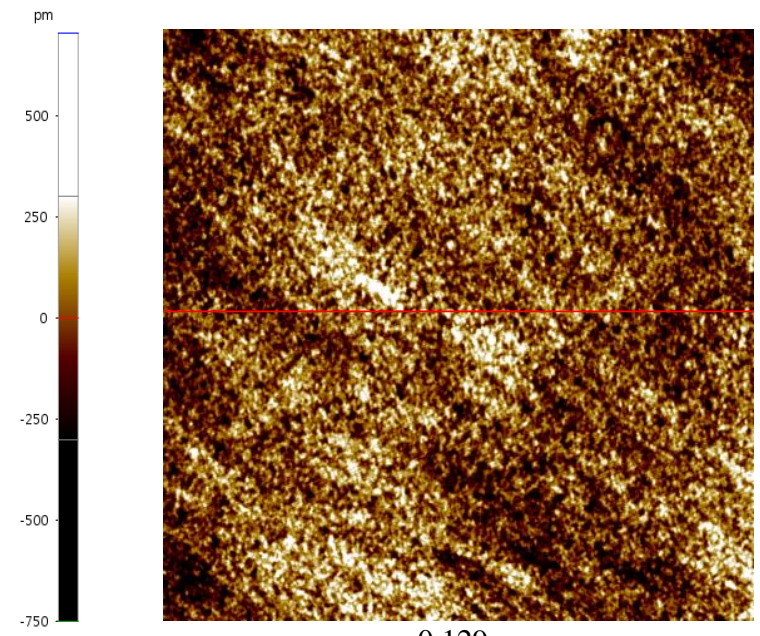

$\sigma=0.129 \mathrm{~nm}$

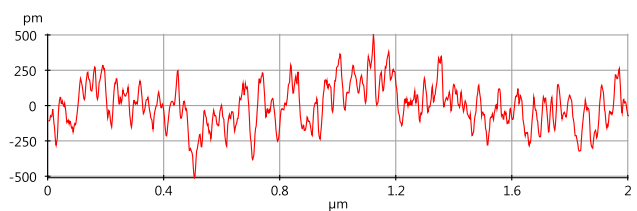

(b)

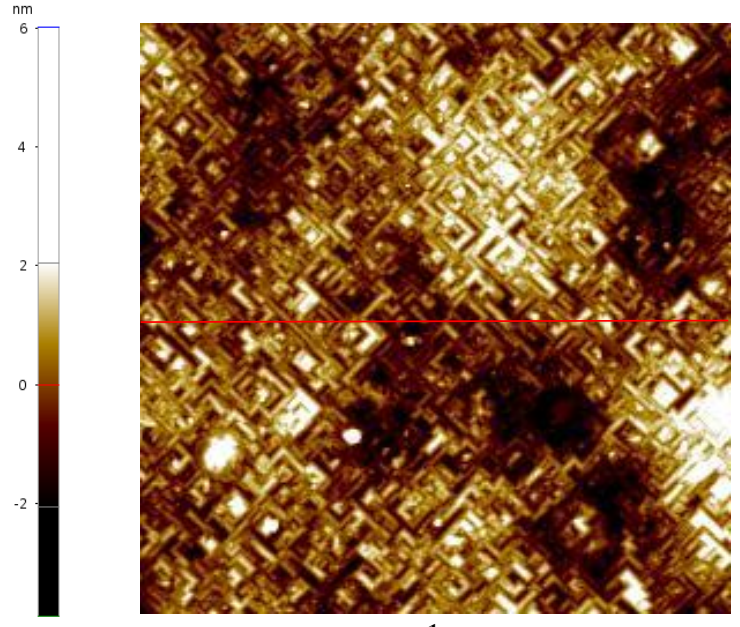

$\sigma=1 \mathrm{~nm}$

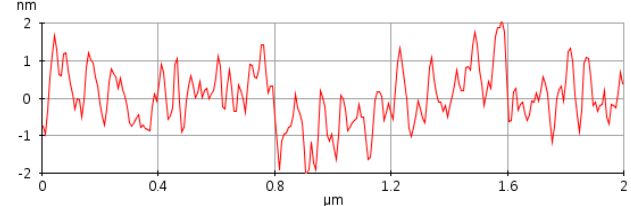

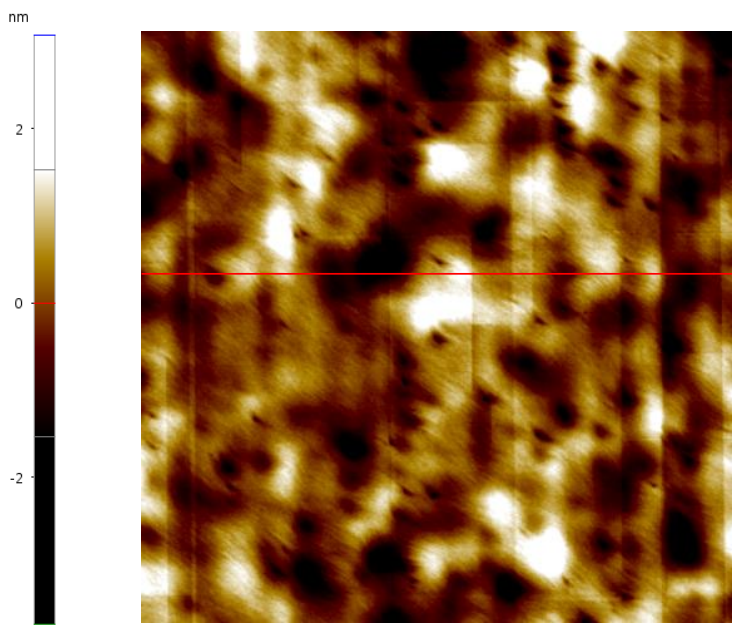

$\sigma=0.77 \mathrm{~nm}$
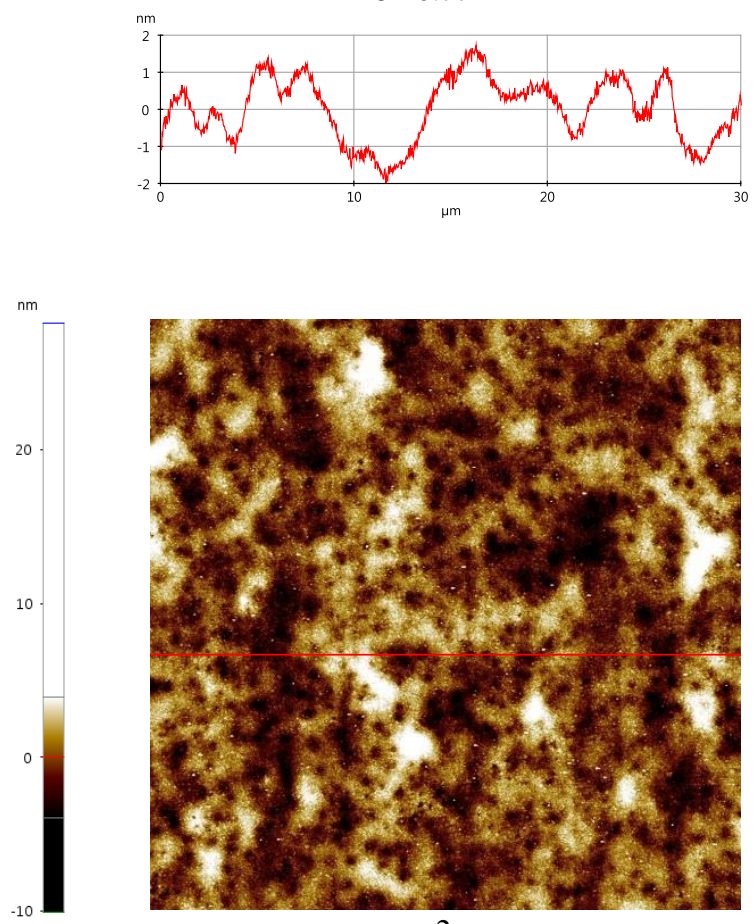

$\sigma=2 \mathrm{~nm}$

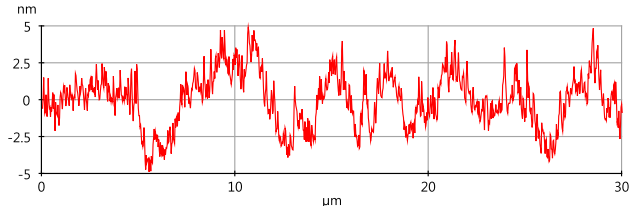

Figure 3. AFM topography images $(2 \times 2$ and $30 \times 30 \mu \mathrm{m})$ and line profiles of $\mathrm{Ge}(100) / \mathrm{Si}(100)$ (a) and $\mathrm{Gr} / \mathrm{Ge}(100) / \mathrm{Si}(100)(\mathrm{b}) \mathrm{surfaces}$. 
The AFM topography scan of $2 \times 2 \mu \mathrm{m}$ for graphene in Figure $3 \mathrm{~b}$ shows that graphene-induced Ge faceting is clearly recognizable as hill-and-valley structures with four-fold symmetry of patterns and a mean square roughness $\sigma$ of $\sim 1 \mathrm{~nm}$. The dimensions of the faceted structure visible in AFM images are typically less than $2 \mathrm{~nm}$ height and nearly $100 \mathrm{~nm}$ wide. The formation of Ge facets during graphene growth by CVD was reported recently [11]. These Ge facets formed at elevated temperatures are stable under ambient conditions after rapid cooling to room temperature as a result of the mismatch of the thermal expansion coefficient between graphene and Ge. At lower temperatures, the germanium diffusion length during the cooling period is short leading to the formation of terraces rather than to a planar (001) surface. Different Ge faceting modifications can be observed with a different cooling rate.
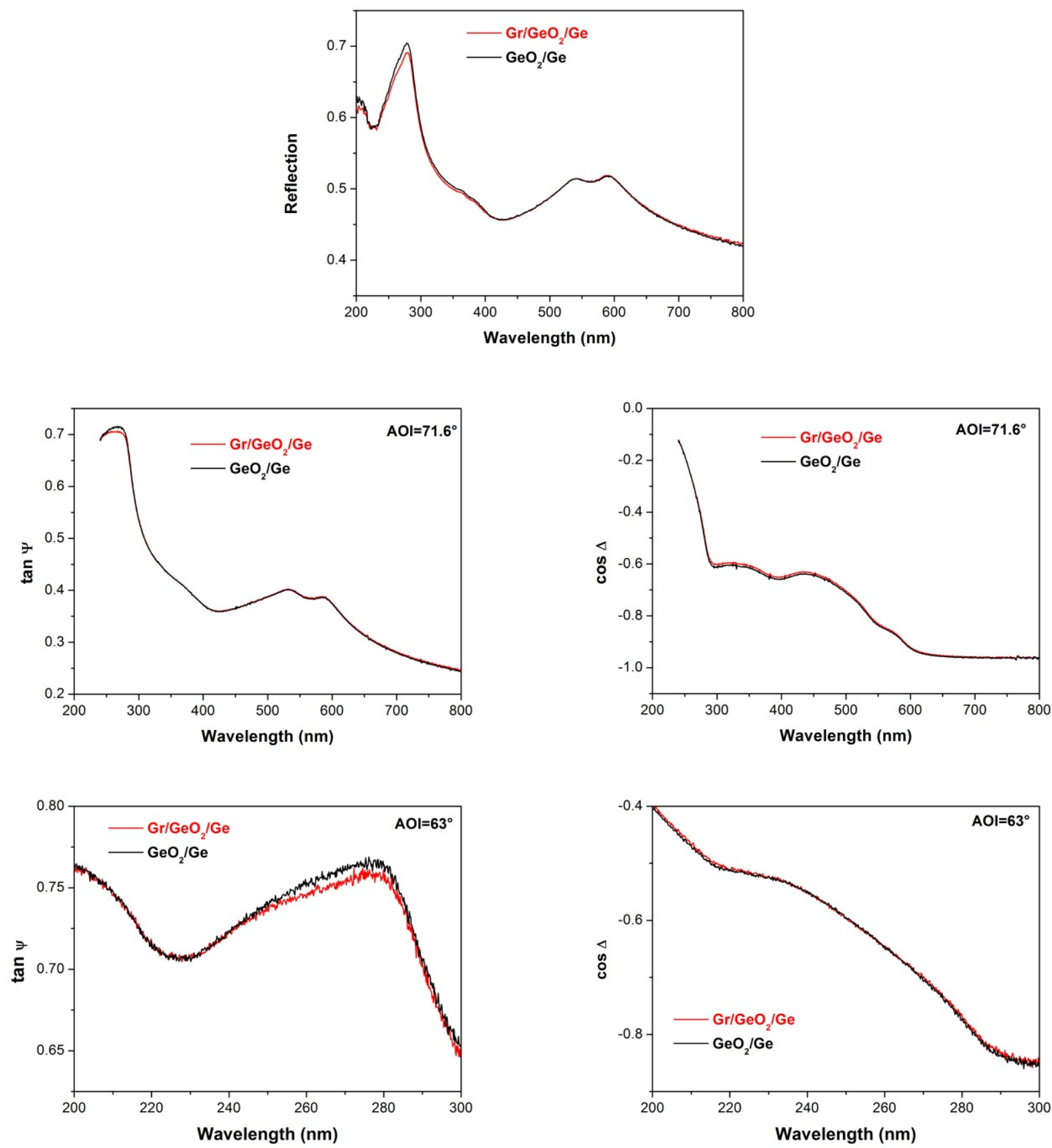

Figure 4. Experimental spectroscopic ellipsometry and reflectometry measurements with and without graphene on $\mathrm{GeO}_{2}(1.1 \mathrm{~nm}) / \mathrm{Ge}(100) / \mathrm{Si}(100)$. 
Industrial implementations of optical metrology setups typically average over large areas by illumination spots of $\sim 30 \mu \mathrm{m}$ and relatively low resolution. That is why a comparable scan size $(30 \mu \mathrm{m})$ was additionally chosen for AFM measurements. The roughness $\sigma$ observed for $30 \mu \mathrm{m}$ scan of graphene surface is $\sim 2 \mathrm{~nm}$ (Figure $3 \mathrm{~b}$ ).

Figure 4 shows the spectroscopic ellipsometry and reflectometry measurements for $\mathrm{GeO}_{2}(1.1 \mathrm{~nm}) / \mathrm{Ge}(100) /$ $\mathrm{Si}(100)$ and graphene on $\mathrm{GeO}_{2}(1.1 \mathrm{~nm}) / \mathrm{Ge}(100) / \mathrm{Si}(100)$ substrates. It is possible to observe small changes in all optical quantities (reflection, $\tan \psi, \cos \Delta$ ) after graphene deposition. Considering also the increase of surface roughness after graphene deposition, as it was observed by AFM (Figure 3), we have modeled both effects in order to find out their impact on optical quantities. The optical quantities (reflection, ellipsometric ratio, scattering) of randomly rough surfaces are dependent on vertical and lateral dimensions of the irregularities. Accurate simulation of the responses in optical quantities and their change due to surface roughness requires experimental statistical information such as root mean square roughness $\sigma$, autocovariance (ACV) function, and autocorrelation length $\left(\mathrm{L}_{\mathrm{c}}\right)$. The main spatial wavelength interval or $\mathrm{L}_{\mathrm{c}}$ is determined, which describes the periodical part of surface roughness, is calculated as 1/e half-width of the ACV function (Figure 5) and for the $30 \mu \mathrm{m}$ scan of $\mathrm{Gr}$ on $\mathrm{Ge}(100) / \mathrm{Si}(100) \mathrm{L}_{\mathrm{c}} \sim 0.7 \pm 0.16 \mu \mathrm{m}$. The spatial wavelengths are comparable with the wavelength of incident light.

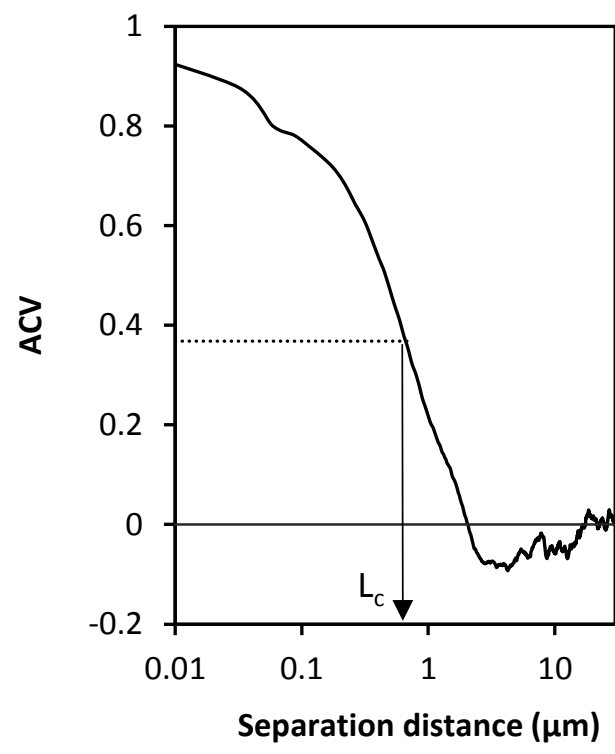

Figure 5. ACV function of $30 \mu \mathrm{m}$ scans and the correlation length $\mathrm{L}_{\mathrm{c}}$ at $\mathrm{ACV}=1 / \mathrm{e}$ for graphene on $\mathrm{Ge}(100) / \mathrm{Si}(100)$ from Figure $3 \mathrm{~b}$.

The theoretical approaches based on the scalar diffraction theory (SDT) [12-14] and the rigorous coupled wave analysis (RCWA) [15] are utilized for the modelling of the surface roughness.

The simulation of the scattering based on SDT is performed using the equation $R=R_{0} \exp \left[-\left(4 \pi \sigma \mathrm{n}_{0} \cos \alpha / \lambda\right)^{2}\right]$ where $\mathrm{R}_{0}$ is the reflection from the plane surfaces, $\sigma$ - the RMS roughness, $\mathrm{n}_{0}$ - the refraction index of the environment and $\alpha$ is AOI. In addition to the smooth surface requirement $(\sigma / \lambda<<1)$, for SDT approach a Gaussian surface height distribution is assumed and that most of the scattered light is restricted to angles close to the specular beam.

RCWA solves the linear Maxwell's equation based on the Fourier expansion of the dielectric function and the electromagnetic fields inside the periodic structure under conditions of $\mathrm{p} / \lambda<10$ and $\mathrm{h} / \mathrm{p} \leq 1$, where $\mathrm{p}$ and $\mathrm{h}$ are period and height of the surface irregularities. For simplification, we have defined $p=2 \mathrm{~L}_{c}$ and $h=2 \sigma$.

According to RCWA, only the main spatial wavelength interval $\left(\mathrm{L}_{\mathrm{c}} \sim 0.7 \pm 0.16 \mu \mathrm{m}\right)$ can contribute to the scattering and can impact on reflection, $\tan \psi$ and $\cos \Delta$. For the graphene faceting (Figure $3 b$ ) where $\mathrm{L}_{c}<<\lambda$ the scattering effect can be neglected. Therefore the optical measurements provide the averaged area information $(\sim 30 \mu \mathrm{m})$ without high spatial resolution.

Simulated results of the reflection, $\tan \psi$ and $\cos \Delta$ under consideration of surface roughness on $\mathrm{Gr} / \mathrm{GeO}_{2} / \mathrm{Ge}$ by different approaches in comparison with smooth $\mathrm{GeO}_{2} / \mathrm{Ge}$ and the corresponding sensitivity plots are shown in Figures 6-9. A simple model of the reflecting system consist of the following three layers: (1) Ge substrate; (2) interlayer of 
native oxide $\left(\mathrm{GeO}_{2}\right)$ which is typically present on the surface of Ge and an indirectly included interface roughness and (3) the deposited layer of graphene. The ambient was air. Optical parameters for $\mathrm{Ge}$ and $\mathrm{GeO}_{2}$ were taken from [16]. All layers were treated isotropically. Si was not included to the optical model due to relative thick Ge layer above which is considered as bulk in UV-Vis spectral range.

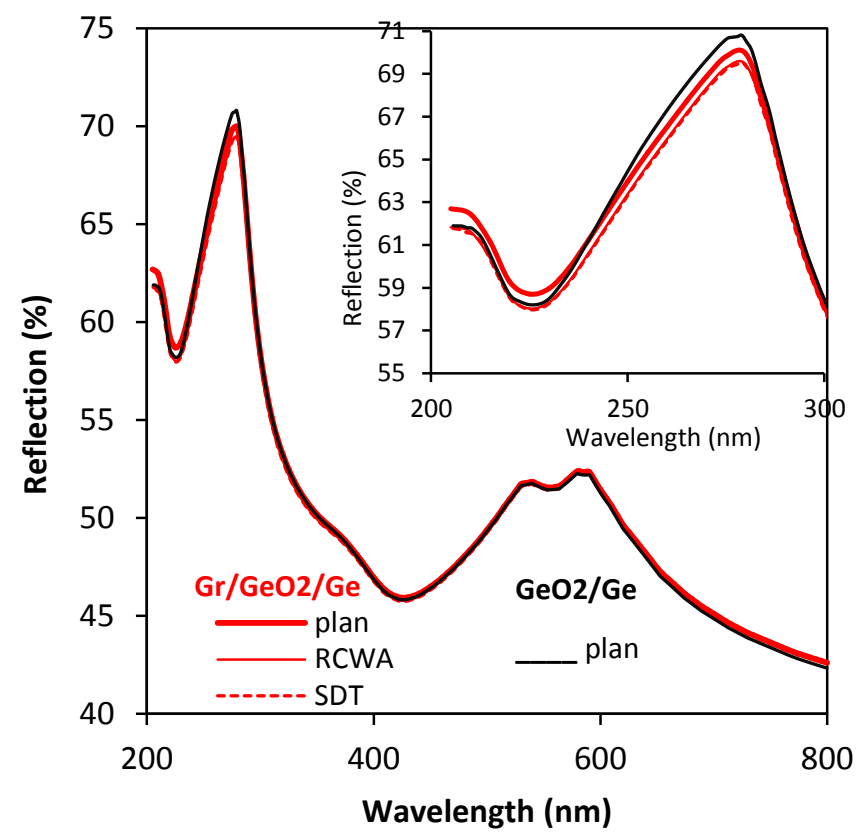

Figure 6. Simulation of the reflection for the plane $\mathrm{GeO}_{2} / \mathrm{Ge}$ and $\mathrm{Gr} / \mathrm{GeO}_{2} / \mathrm{Ge}$ with surface roughness modeled with SDT and RCWA approaches.

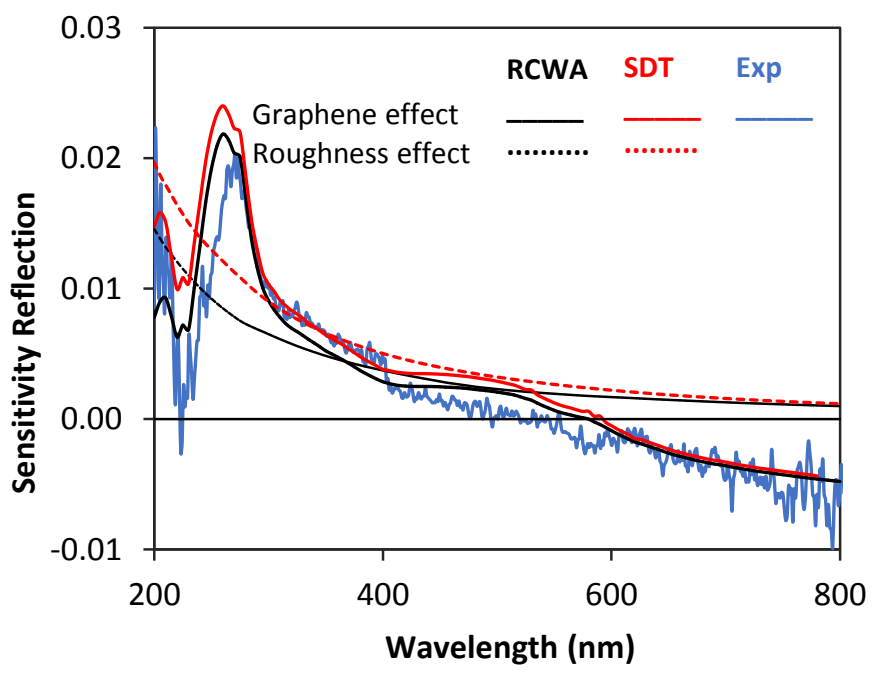

Figure 7. Experimental (blue) and simulated (red, black) sensitivities of the reflections for the demonstration of the monolayer graphene deposition on $\mathrm{Ge}(100) / \mathrm{Si}(100)$ in comparison with roughness effect calculated by RCWA and SDT approaches. The simulated sensitivities $\left(R_{\text {plan }}-R_{\sigma}\right) / R_{\text {plan }}$ show the roughness effect, where $R_{\text {plan }}$ and $R_{\sigma}$ are the reflections for the plane and with surface roughness $(\sigma=2 \mathrm{~nm})$ models of $\mathrm{Gr} / \mathrm{GeO}_{2} / \mathrm{Ge}$. The experimental and simulated sensitivities $\left(\mathrm{R}_{\mathrm{Sub}}-\mathrm{R}_{\sigma}\right) / \mathrm{R}_{\text {Sub }}$ show the possibilities of monolayer graphene on $\mathrm{GeO}_{2} / \mathrm{Ge}$ substrate detection. 

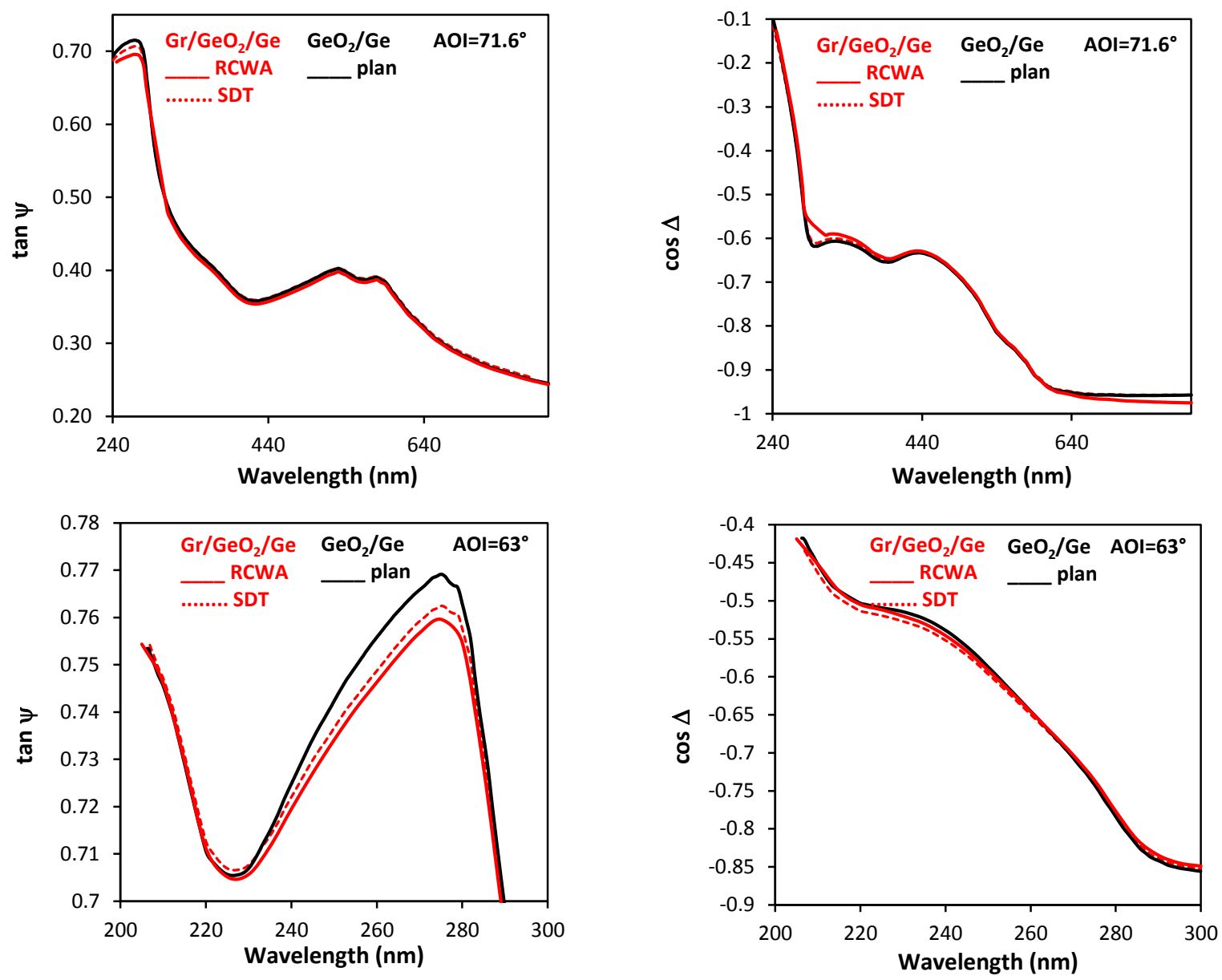

Figure 8. Simulation of ellipsometric parameters tan $\psi$ and $\cos \Delta$ for plane $\mathrm{GeO}_{2} / \mathrm{Ge}$ and $\mathrm{Gr} / \mathrm{GeO}_{2} / \mathrm{Ge}$ with surface roughness modeled with SDT and RCWA approaches performed for AOIs of experimental setup (71.3 and $\left.63.04^{\circ}\right)$.
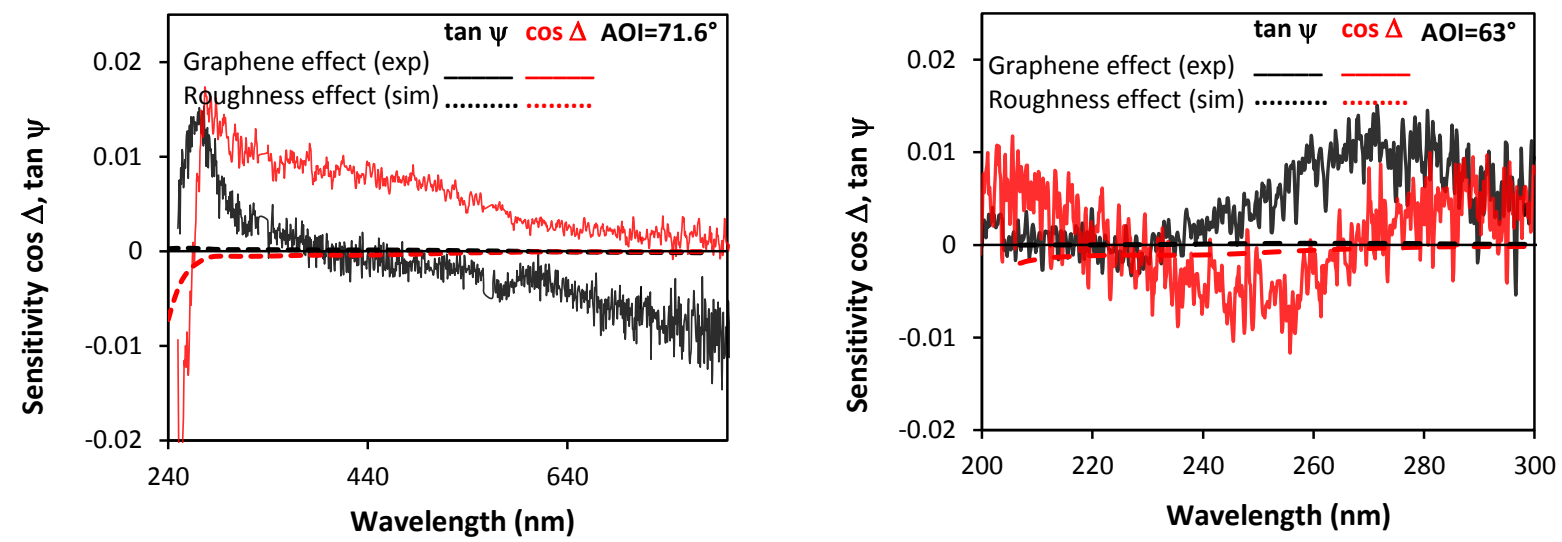

Figure 9. Experimental and simulated sensitivities of $\tan \psi$ and $\cos \Delta$ for the demonstration of the monolayer graphene deposition on $\mathrm{Ge}(100) / \mathrm{Si}(100)$ in comparison with roughness effect calculated by the SDT approach for AOIs of experimental setup (71.3 and $\left.63.04^{\circ}\right)$. The simulated sensitivities $\left(\tan \psi_{\text {plan }}-\tan \psi_{\sigma}\right) / \tan \psi_{\text {plan }}$ and $\left(\cos \Delta_{\text {plan }}-\cos \Delta_{\sigma}\right) / \cos \Delta_{\text {plan }}$ show the roughness effect, where $\cos \Delta_{\text {plan }}$, $\tan \psi_{\text {plan }}$ correspond to the plane model and $\cos \Delta_{\sigma}$, $\tan \psi_{\sigma}$ correspond to the model with surface roughness $(\sigma=2 \mathrm{~nm})$ of $\mathrm{Gr} / \mathrm{GeO} / 2 \mathrm{Ge}$. The experimental sensitivities $\left(\tan \psi_{\text {Sub }}-\tan \psi_{\sigma}\right) / \tan \psi_{\text {Sub }}$ and $\left(\cos \Delta_{\text {Sub }}-\cos \Delta_{\sigma}\right) / \cos \Delta_{\text {Sub }}$ show the possibility of monolayer graphene on $\mathrm{GeO}_{2} / \mathrm{Ge}$ substrate detection. 
The main conclusion resulting from Figures 6-9 is that the effect of surface roughness has much smaller influence on all optical quantities, i.e. reflection, $\tan \psi$ and $\cos \Delta$ than the effect of monolayer graphene deposition. The RCWA as well as SDT approach including roughness effects which were applied for the ellipsometry simulations deliver approximately the same results as the plane model. Thus, the spectroscopic ellipsometry measurements have no sensitivity to the roughness of graphene. Otherwise, the existence of surface roughness has impact on the reflectometry. The main reason is the low depolarization associated with a relatively low roughness. Furthermore, the ellipsometry in contrast to the reflectometry is a relative measuring method. Therefore, it is also possible to neglect the effect of surface roughness for structure modelling in order to detect the graphene monolayer on $\mathrm{GeO}_{2} / \mathrm{Ge}$ with a simple and robust procedure by ellipsometry.

Considering the reflection sensitivity plot (Figure 7) we can conclude that reflectometry alone cannot provide enough sensitivity for adequate determination of monolayer graphene. The sensitivity to graphene monolayer is quite small and only visible in UV range, where the noises in reflectance measurements and surface roughness have similar effect on the reflection.

The sensitivity of ellipsometry measurements (Figure 9) shows the responses of both $\tan \psi$ and $\cos \Delta$ on the existence of graphene monolayer, especially $\cos \Delta$ for $\mathrm{AOI}=71.6^{\circ}$. In the case of $\mathrm{AOI} \approx 63^{\circ}$ the $\tan \psi$ has higher sensitivity in 250-300 nm range. Maximum sensitivity of the spectroscopic ellipsometry setup is observed in the ultraviolet region of the spectrum $(250-400 \mathrm{~nm})$ where a strong peak of the extinction coefficient of graphene is identified and a difference up to $2.0 \%$ between the bare substrate and graphene on the substrate is observed. The change in $\Delta$ is about $1.5^{\circ}$ at 300 $\mathrm{nm}$ for the graphene monolayer and well over an order of magnitude higher than the sensitivity limit of the tool $\left(\sim 0.02^{\circ}\right)$. Additionally 600-800 $\mathrm{nm}$ wavelength range can improve the sensitivity for graphene monolayer identification where a higher sensitivity of $\tan \psi$ is observed. Such sensitivity makes graphene surely detectable and measurable on $\mathrm{Ge}(100) / \mathrm{Si}(100)$ substrates. Therefore, ellipsometry setup can be a suitable choice for graphene layer evaluation.

Summarized, combined data collection (ellipsometry and reflectometry) facilitates unambiguous analysis of graphene on Si for complex evaluation of a graphene layer stack, including the surface roughness, especially when the irregularities of the surface are small compared with the wavelength of incident light but higher than thickness of corresponding layer.

A robust recipe for unambiguous thickness monitoring of all components of a multilayer film stack, including graphene, surface residuals or interface layer underneath graphene and surface roughness is developed and applied for process control of CVD graphene on $200 \mathrm{~mm} \mathrm{Ge}(100) / \mathrm{Si}(100)$ wafers. Figure 10 shows maps of 225 points for graphene, $\mathrm{GeO}_{2}$ thicknesses and surface roughness. The measured average graphene thickness was $\sim 0.34 \mathrm{~nm}$ with a standard deviation value of $4 \%$ over the full wafer. This indicates a homogeneous distribution of graphene over the wafer and is in a good agreement with Raman measurements. Optical monitoring of the graphene thickness uniformity over a wafer has shown an excellent long term stability $(s=0.004 \mathrm{~nm})$ during 4 month evaluation time independent on growth of interfacial $\mathrm{GeO}_{2}$ and surface roughness.

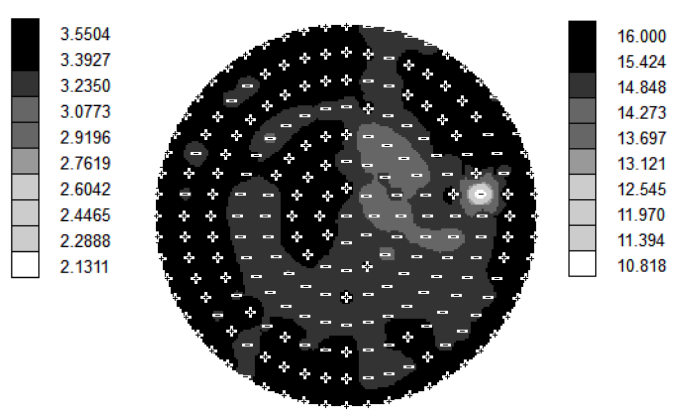

(a)

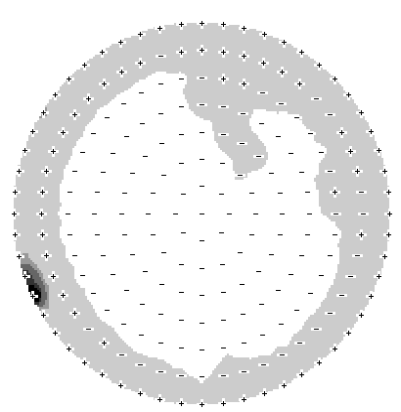

(b)

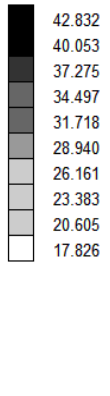

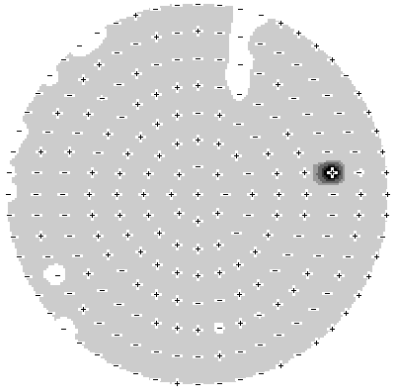

(c)

Figure 10. Thickness maps of graphene (a), $\mathrm{GeO}_{2}$ (b) and surface roughness (c) in $\AA$ for graphene/GeO $/ 2 \mathrm{Ge}(100) / \mathrm{Si}(100) 200-\mathrm{mm}$ wafer. The marked sites correspond to the measurement positions. 


\section{CONCLUSION}

A fast and nondestructive metrology method is demonstrated which allows one to performed a reliable identification of graphene after the CVD graphene deposition process on $200-\mathrm{mm} \mathrm{Ge}(100) / \mathrm{Si}(100)$ wafer. The method is based on a combination of UV-Vis spectroscopic ellipsometry and near normal incidence reflectometry with small light spots $(\sim 30$ $\mu \mathrm{m}^{2}$ ), realized in a wafer optical metrology tool. Optical constants for graphene were accurately determined on $\mathrm{SiO}_{2} / \mathrm{Si}$ substrates using broadband multi-angle reflectometry-ellipsometry techniques. The effect of surface roughness and graphene monolayer were evaluated concerning their influence on the optical quantities (reflection, $\tan \psi, \cos \Delta$ ).

A robust recipe for unambiguous thickness monitoring of all components of a multilayer film stack, including graphene, surface residuals or interface layer underneath graphene and surface roughness is developed and applied for

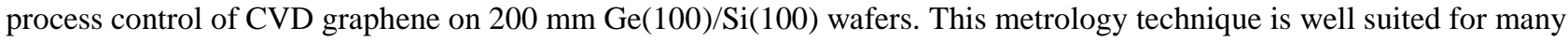
industrial applications due to its repeatability and flexibility.

\section{REFERENCES}

1. Li X, Cai W, An J., Kim S., Nah J., Yang D. et al, "Large-area synthesis of high-quality and uniform graphene films on copper foil," Science 324 (5932), 1312-4 (2009).

2. Pasternak I., Wesolowski M., Jozwik I., Lukosius M., Lupina G., Dabrowski P., Baranowski J., Strupinski W., "Graphene growth on Ge(100)/Si(100) substrates by CVD method," Scientific Reports 6, 121773 (2016).

3. Pasternak I., Dabrowski P., Ciepielewski P., Kolkovsky V., Klusek Z., Baranowski J.M., Strupinski W., "Largearea high-quality graphene on Ge(001)/Si(001) substrates," Nanoscale 8, 11241-11247 (2016).

4. Yamamoto Y., Zaumseil P., Arguirov T., Kittler M., Tillack B., "Low threading dislocation density Ge deposited on Si(100) using RPCVD," Solid-State Electronics 60, 2-6 (2011).

5. Gray A., Balooch M., Allegret S., De Gendt S., Wang Wei-E, "Optical detection and characterization of graphene by broadband spectrophotometry,” Journal of Appl. Phys. 104, 053109 (2008).

6. Lupina G., Lukosius M., Lippert G., Dabrowski J., Kitzmann J., Lisker M., Kulse P., Krueger A., Fursenko O., Costina I., Trusch A., Yamamoto Y., Wolff A., Schroeder T., Mai A., "Graphene synthesis and processing on Ge substrates,” ECS J. Solid State Sci. Technol. 6, M55-M59 (2017).

7. Lukosius M., Dabrowski J., Kitzmann J., Fursenko O., Akhtar F., Lisker M., Lippert G., Schulze S, Yamamoto Y., Schubert M. A., Krause H. M., Wolff A., Mai A., Schroeder T, Lupina G., "Metal-free CVD graphene synthesis on $200 \mathrm{~mm} \mathrm{Ge/Si} \mathrm{(100)} \mathrm{substrates,"} \mathrm{ACS} \mathrm{Appl.} \mathrm{Mater.} \mathrm{Interfaces} \mathrm{8(49),} \mathrm{33786-33793} \mathrm{(2016).}$

8. Lupina G., Kitzmann J., Costina I., Lukosius M., Wenger Ch., Wolff A., Vaziri S., Ostling M., Pasternak I., Strupinski W., Kataria S., Gahoi A., Lemme M.C., Ruhl G., Zoth G., Luxenhofer O., Mehr W., "Residual Metallic Contamination of Transferred Chemical Vapor Deposited Graphene," ACS Nano 9, 4667 (2015).

9. Kravets V. G., Grigorenko A.N., Nair R. R., Blake P., Anissimova S., Novoselov K. S., Geim A. K., "Spectroscopic ellipsometry of graphene and an exciton-shifted van Hove peak in absorption," Phys. Rev. B 81, 155413 (2010).

10. Boosalis A., Elmquist R., Real M., Nguyen N., Schubert M., and Hofmann T., "A model dielectric function for graphene from the infrared to the ultraviolet," Mater. Res. Soc. Symp. Proc. 1505 (2013).

11. McElhinny K.M., Jacobberger R.M., Zaug A.J., Arnold M.S., Evans P.G., "Graphene-induced Ge (001) surface faceting," Surface Science 647, 90-95 (2016).

12. Davies H., "The reflection of electromagnetic waves from a rough surface," Proc. IEEE Part IV 101, 209-214 (1954).

13. Beckmann, P. and A. Spizzichino, "The Scattering of Electromagnetic Waves from Rough Surfaces," Pergamon, New York (1963).

14. Ohlidal I., Navratil K., Ohlidal M., "Scattering of light from multilayer systems with rough boundaries," in E. Wolf (Ed.), Progress in Optics 34, 249-331 (1995).

15. http://www.unigit.com

16. Palik E.D. (ed), Handbook of Optical Constants of Solids, Academic Press, San Diego (1991). 